\title{
Correlation Between Anthropometric Measurements and Sensory Block Level of Spinal Anesthesia for Cesarean Section
}

\author{
Hyojoong Kim ${ }^{1,}{ }^{*}$, Sung Hyun Shin ${ }^{1}$, Myoung Jin Ko $^{1}$, Yei Heum Park ${ }^{1}$, Ki Hwa Lee ${ }^{1}$, Kyung-Hoon Kim ${ }^{2}$ \\ and Tae-Kyun Kim ${ }^{2, * *}$ \\ ${ }^{1}$ Department of Anesthesiology and Pain Medicine, Haeundae Paik Hospital, Inje University, Busan, South Korea \\ ${ }^{2}$ Department of Anesthesia and Pain Medicine, Pusan National University School of Medicine, Pusan National University Yangsan Hospital, Yangsan, South Korea \\ "Corresponding author: Department of Anesthesiology and Pain Medicine, Haeundae Paik Hospital, Inje University, Busan, South Korea. Email: ho0386@paik.ac.kr \\ ${ }^{* *}$ Corresponding author: Department of Anesthesia and Pain Medicine, Pusan National University School of Medicine, Pusan National University Yangsan Hospital, Yangsan, \\ South Korea. Email: anesktk@pusan.ac.kr
}

Received 2021 August 11; Revised 2021 October 07; Accepted 2021 October 07.

\begin{abstract}
Background: When performing spinal anesthesia for cesarean section, it is important to determine the appropriate anesthetic dose as well as to predict the level of spinal anesthesia. In this study, it was hypothesized that some anthropometric measurements may be related to maximum sensory block and hemodynamic changes.

Objectives: The aim of this study are to find maternal anthropometric values that are correlate with the level of spinal anesthesia. Methods: Maternal anthropometric measurements, including height, weight, supine and standing abdominal circumference (AC), and hip circumference, were recorded before spinal anesthesia for cesarean section. Spinal anesthesia was induced by administering $8 \mathrm{mg}$ of $0.5 \%$ hyperbaric bupivacaine and $20 \mu \mathrm{g}$ of fentanyl at the L3-L4 interspace. The level of sensory block was determined using pin-prick at 1, 5, 10, and 15 minutes after spinal anesthesia. The sensory block level and hemodynamic adverse events were analyzed in relationship to anthropometric measurements.

Results: The supine AC/height ratios significantly correlate with the maximal sensory block level at 5,10, and 15 minutes after the injection of spinal anesthetic $(\mathrm{P}=0.001, \mathrm{P}<0.001$ and $\mathrm{P}<0.001$, respectively). Further, there were significant correlations between body mass index (BMI) and sensory block level at every assessment $(\mathrm{P}=0.041, \mathrm{P}=0.002, \mathrm{P}=0.001$ and $\mathrm{P}<0.001$, respectively). When comparing the groups with and without hypotension, $\mathrm{BMI}$, weight, and supine AC/height ratio were found to be significantly higher in the group with hypotension $(\mathrm{P}=0.002, \mathrm{P}=0.004$ and $\mathrm{P}=0.006$, respectively).

Conclusions: We conclude that BMI and AC/height ratio correlate with the sensory block level of spinal anesthesia for cesarean section.
\end{abstract}

Keywords: Anesthetics, Anthropometric Measurements, Bupivacaine, Cesarean Section, Fentanyl, Hemodynamics, Hypotension, Sensory Block, Spinal Anesthesia

\section{Background}

Spinal anesthesia is one of the most frequently used anesthetic methods, along with general anesthesia, especially for cesarean section. The main reason of frequent use is that it is associated with fewer airway-related complications compared to general anesthesia. In addition, the fetus is not exposed to anesthetics, the mother can remember the experience, and it is excellent in managing pain after surgery $(1,2)$.

The factors affecting the level of spinal anesthesia include the patient's height, weight, site of injection, and pregnancy (3). In particular, the level of spinal anesthesia increases even when the same dose of anesthetic is administered due to increased intra-abdominal pressure in the parturient (4). Moreover, increase in neural sensitivity makes the parturient sensitive to local anesthetics and changes in the lumber lordosis increase the physical spread of the solution; hence, the same level of spinal anesthesia can be achieved with a small dose of anesthetics compared to non-parturients $(5,6)$.

Therefore, when performing spinal anesthesia for cesarean section, apart from selecting an appropriate anesthetic dosage, predicting the level of spinal anesthesia is also important. For the prediction of the level of spinal anesthesia, it is thought that the maternal anthropometric measurements have a significant influence, but there are not many such studies yet, and the results are inconsistent. 


\section{Objectives}

Therefore, the aim of this study are to find out maternal anthropometric values that are more correlate with the level of sensory block than previous studies and to compare that values with the occurrence of side effects.

\section{Methods}

This is an observational study, approved by the Institutional Review Board of Haeundae Paik Hospital (HPIRB2017-09-001-007). Patients were included who were undergoing elective cesarean section, aged 20-40 years, and over 36 weeks of singleton gestation. A total of 50 patients were included. Informed consents were obtained from all the patients the night before surgery. The exclusion criteria were patient rejection, contraindication to spinal, preeclampsia, eclampsia, twin pregnancy and failed spinal anesthesia.

All patients' height, weight, pre-pregnancy weight, body mass index (BMI), gestational weeks, and ultrasound fetal weight were recorded. One researcher measured the abdominal circumference (AC) at the height of the umbilicus in the standing and supine positions and the hip circumference (HC) at the protruding part of the buttocks in the standing. In the operating room, blood pressure, pulse oximetry and electrocardiography were monitored, and the patients were infused rapidly with $500 \mathrm{ml}$ of balanced solution. While infusing the solution, patients were asked to turn to the left lateral decubitus position. Spinal anesthesia was performed at the L3/4 level with a 24G. Whitacre needle by the median approach. We injected a mixture of $0.5 \%$ hyperbaric bupivacaine (Bupivacaine $\mathrm{HCl} \circledast$, Hana Pharm, South Korea) $8 \mathrm{mg}$ and fentanyl $20 \mu \mathrm{g}$. After spinal anesthesia, the patients were returned to the supine position, and the bed was tilted to the left side for uterine displacement. We recorded the blood pressure and heart rate at 1-minute intervals for 5 minutes, followed by 2-minute intervals for 10 minutes.

Hypotension was defined as a mean blood pressure lower than $65 \mathrm{mmHg}$ or a decrease of more than 30\% from the baseline blood pressure. A bolus of phenylephrine 0.05 mg was administered when hypotension occurred. Abolus of atropine $0.5 \mathrm{mg}$ was administered when the heart rate was less than 50 bpm without hypotension. Sensory blockade level was monitored using a pinprick test at 1, 5, 10, and 15 minutes after spinal anesthesia.

Sample size was based on a previous study. Primary outcome was determined as the relationship between anthropometric measurements and sensory block after spinal anesthesia. Based on the study by Baysal et al. (7), the correlation coefficient was expected to be 0.4. A power analysis $(\mathrm{a}=0.05, \mathrm{~b}=0.20)$ showed that a total of $46 \mathrm{pa}$ tients would be required; considering $10 \%$ of dropout, 50 patients were enrolled.

All statistical analyses were performed using SPSS Statistics 25.0 (IBM Corporation, Armonk, NY), and p values less than 0.05 were considered statistically significant. The data are presented as frequency with a noted percentage for categorical variables and mean \pm standard deviation for continuous variables. The Spearman correlation coefficient was used to determine the association between the anthropometric measurements and level of sensory block. The differences in other characteristics were compared using the chi-squared test or Fisher's exact test for categorical variables, and independent t-test or Mann-Whitney U test for continuous variables, as appropriate.

\section{Results}

No patients were excluded out of the 50 participants enrolled in this study. Age, anthropometric values, gestational weeks, and fetal body weight are shown in Table 1.

\begin{tabular}{lc}
\hline \multicolumn{2}{l}{ Table 1. Demographic Data and Anthropometric Data of Patients } \\
\hline Variables & Mean \pm SD \\
\hline Age $(\mathbf{y})$ & $34.2 \pm 4.0$ \\
\hline Height $(\mathbf{c m})$ & $162.1 \pm 6.3$ \\
\hline BMI before pregnant $\left(\mathbf{k g} / \mathbf{c m}^{\mathbf{2}}\right)$ & $22.9 \pm 3.8$ \\
\hline BMI after pregnant $\left(\mathbf{k g} / \mathbf{c m}^{2}\right)$ & $27.6 \pm 3.6$ \\
\hline Fetal gestational age $(\mathbf{w e e k})$ & $37.9 \pm 0.7$ \\
\hline Baby body weight $(\mathbf{g})$ & $2944.4 \pm 379.7$ \\
\hline AC $(\mathbf{s t a n d i n g})(\mathbf{c m})$ & $104.9 \pm 6.9$ \\
\hline AC $(\mathbf{s u p i n e})(\mathbf{c m})$ & $102.7 \pm 6.5$ \\
\hline HC $(\mathbf{c m})$ & $104.1 \pm 7.3$ \\
\hline
\end{tabular}

Abbreviations: AC, abdominal circumference; BMI, body mass index; HC, hip circumference.

On analyzing the correlation between the patients' anthropometric values and the level of spinal anesthesia, it was found that the greater the supine AC, the higher the level of spinal anesthesia reached at 5, 10, and 15 minutes after the injection of spinal anesthetic $(\mathrm{P}<0.05)$. Further, supine AC per height and standing AC per height were correlated with the level of spinal anesthesia at 5, 10, and 15 minutes after the injection $(\mathrm{P}<0.05)$. The BMI was correlated with the level of spinal anesthesia at all measurement points $(\mathrm{P}<0.05)$, and the correlation co-efficiency at 15 minutes after spinal anesthesia was also the greatest $(r=$ $0.51, \mathrm{P}<0.001$ ) (Table 2). There was no correlation between $\mathrm{AC}$ per $\mathrm{HC}$ and the level of spinal anesthesia. 


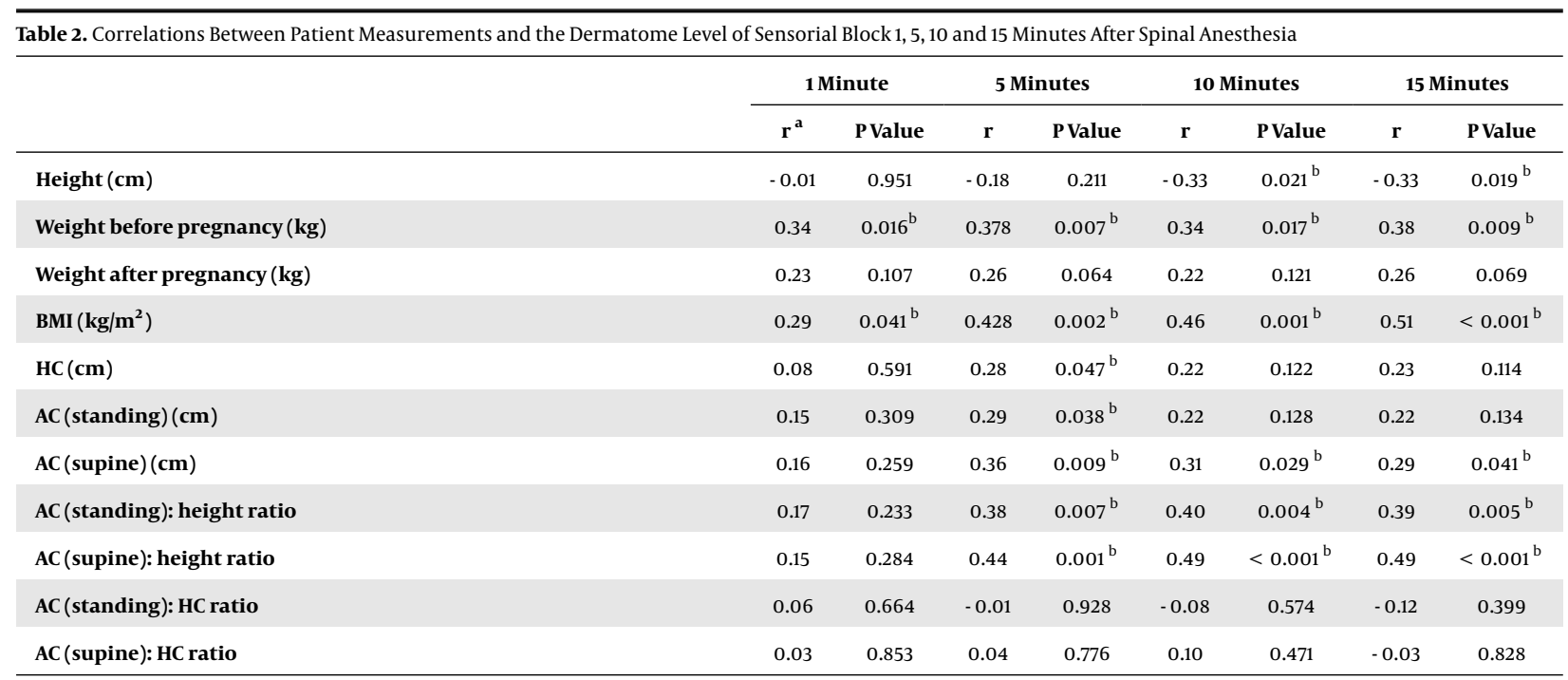

Abbreviations: AC, abdominal circumference; BMI, body mass index; HC, hip circumference

a $r$ : Spearman correlation coefficient.

${ }^{\mathrm{b}} \mathrm{P} \leq 0.05$ means statistically significant.

The most frequent highest level of spinal anesthesia was at the T4 dermatome (24\%). Among all the subjects, the highest level of spinal anesthesia was at the T2 dermatome, and the lowest level of spinal anesthesia was at the T10 dermatome.

Hypotension was observed in 26 patients (52\%). When comparing the anthropometric measurements between groups with and without hypotension, current weight, pre-pregnancy weight, BMI, HC, supine AC, and supine AC per height were significantly higher in the group without hypotension (Table 3).

\section{Discussion}

The purpose of this study was to find out whether the parturients' physical characteristics were related to the level of spinal anesthesia. There were several studies that compared the characteristics of the parturient and the level of spinal anesthesia, but no consistent results were obtained. Norris (8) compared the level of spinal anesthesia and height, weight, and height of the vertebral column, but no variables showed significant correlation. Another study reported that the maximum level of spinal anesthesia was positively correlated with AC, BMI, and weight and negatively correlated with height, body surface area, and $\mathrm{AC} / \mathrm{HC}$ ratio (7). Many anthropometric values were used to reveal the correlation with the level of spinal anesthesia in the current study. The results showed that supine AC, supine $\mathrm{AC} /$ height ratio, and standing $\mathrm{AC} /$ height ratio were correlated with the level of spinal anesthesia at 5,10, and 15 minutes after the injection of anesthetic, and BMI and prepregnancy body weight were positively correlated with the level of spinal anesthesia in all sections.

Among the many factors that affect the level of spinal anesthesia are factors related to patient characteristics including age, height, weight and intra-abdominal pressure (9). Patient characteristics that can change with the progress of pregnancy are weight and intra-abdominal pressure. Based on the literature reports indicating that the level of spinal anesthesia was higher in mothers with twins than in those with a single fetus, it would be expected that there would be a correlation between the degree of weight gain and the level of spinal anesthesia (10). However, Ekelof et al. (11) reported that weight gain was not related with the level of spinal anesthesia, and the same result was obtained in this study. It is known that the weight gain after pregnancy is about $11-15 \mathrm{~kg}$ in normalweight women; however, this also varies greatly between individuals. In addition, weight gain during pregnancy is affected by a number of factors, such as the weight of the fetus, the amount of amniotic fluid, and the volume of plasma. Therefore, it seems unreasonable to use simple weight gain as a factor for predicting the level of spinal anesthesia, since the maternal body composition may vary even with the same weight gain.

The normal intra-abdominal pressure is about 0-5 $\mathrm{mmHg}$, but it is increased to more than $10 \mathrm{mmHg}$ in parturients. If there is a relationship between AC and intraabdominal pressure, it can be used as a predictor of the level of spinal anesthesia, but Malbrain et al. (12) failed 


\begin{tabular}{|c|c|c|c|}
\hline & Hypotension $(\mathbf{n}=26)$, Mean \pm SD & No hypotension $(n=24)$, Mean \pm SD & P Value \\
\hline Height $(\mathrm{cm})$ & $161.8 \pm 6.6$ & $162.5 \pm 6.1$ & 0.717 \\
\hline Weight before pregnancy (kg) & $63.8 \pm 11.2$ & $56.1 \pm 5.9$ & $0.004^{\mathrm{a}}$ \\
\hline Weight after pregnancy $(\mathrm{kg})$ & $76.3 \pm 10.2$ & $68.3 \pm 7.2$ & $0.003^{\mathrm{a}}$ \\
\hline $\operatorname{BMI}\left(\mathrm{kg} / \mathrm{m}^{2}\right)$ & $24.4 \pm 4.4$ & $21.3 \pm 2.1$ & $0.002^{\mathrm{a}}$ \\
\hline $\mathrm{HC}(\mathrm{cm})$ & $106.9 \pm 6.6$ & $100.9 \pm 6.8$ & $0.003^{\mathrm{a}}$ \\
\hline AC (standing) $(\mathrm{cm})$ & $106.4 \pm 6.6$ & $103.1 \pm 6.8$ & 0.087 \\
\hline AC (supine) $(\mathrm{cm})$ & $105.1 \pm 6.8$ & $100.1 \pm 5.2$ & $0.006^{\mathrm{a}}$ \\
\hline AC (standing): height ratio & $0.659 \pm .048$ & $0.635 \pm 0.034$ & 0.066 \\
\hline AC (supine): height ratio & $0.650 \pm .046$ & $0.617 \pm 0.034$ & $0.006^{\mathrm{a}}$ \\
\hline AC (standing): HC ratio & $0.996 \pm .040$ & $1.02 \pm .065$ & 0.071 \\
\hline AC (supine): HC ratio & $0.983 \pm .037$ & $0.995 \pm .060$ & 0.417 \\
\hline
\end{tabular}

Abbreviations: AC, abdominal circumference; BMI, body mass index; HC, hip circumference ${ }^{\mathrm{a}} \mathrm{P} \leq 0.05$ means statistically significant.

to demonstrate this relationship and hence, AC cannot be used as a predictor. And this was consistent with this study. Baysal et al. (7) reported that higher the AC/HC ratio, the higher the level of spinal anesthesia, but the current study showed no such correlation. This is assumed to be the result of the difference in BMI of the parturients in the two studies. They reported that the mean BMI was $30.1 \pm 2.1$ at the time of surgery, but in the current study, the mean BMI was $27.6 \pm 3.6$. The waist/hip ratio is significant in obesity, and it has been reported that a higher waist/hip ratio is related to central obesity and the incidence of metabolic syndrome $(13,14)$. Considering that obese patients showed a higher intra-abdominal pressure than normal-weight patients (15), although Baysal et al. did not provide pre-pregnancy BMI, it is assumed to have been higher than that in this study.

It is well known that obesity is associated with the level of spinal anesthesia. This contributes not only to an increase in intra-abdominal pressure, but also to a narrowing of the epidural space due to the accumulation of fat and an increase in plasma volume (16). According to a previous meta-analysis, not only the BMI and the AC/hip ratio, but also the $\mathrm{AC} /$ height ratio were strong predictors of the occurrence of metabolic syndrome due to obesity (17). In the current study, the pre-pregnancy weight, BMI, and $\mathrm{AC} /$ height ratio showed positive correlations, suggesting that the anthropometric values related to obesity are particularly related to the level of spinal anesthesia.

One of the common complications associated with spinal anesthesia is hypotension. Hypotension occurs as a result of the sympathetic block, and the higher the level of spinal anesthesia, the more likely is its occurrence (18).
In particular, there are many studies which report that maternal hypotension in cesarean section occurs in $25 \%$ or more cases, although there are differences depending on the study (19-21). In the current study, even with intravenous solution, hypotension of less than $70 \%$ of baseline blood pressure occurred in $52 \%$ of patients. Comparing anthropometric measurements in groups with and without hypotension, pre-pregnancy weight, current weight, BMI, and supine $\mathrm{AC} /$ height ratio were significantly higher in groups with hypotension. Therefore, in the case of parturients, the more weight they gain, the greater the chances of hypotension, even if the same dose of anesthetic is administered.

There are some limitations to the present study. First, the same dose of local anesthetic was used for all patients. There are several studies which showed that the use of a local anesthetic dose corrected for height or weight had fewer side effects $(22,23)$. In the current study, neither the mother nor the fetus experienced any serious side effects due to hypotension, but it is presumed that using the corrected doses would further reduce the incidence of hypotension. Second, in predicting the level of spinal anesthesia, we failed to find an anthropometric value that is superior to the BMI. If we try more combinations of anthropometric values, we may find a variable with a stronger correlation.

In conclusion, $\mathrm{BMI}$ and $\mathrm{AC} /$ height ratio were found to be correlated the level of spinal anesthesia at 5,10, and 15 minutes after the injection of spinal anesthetic. And the correlation coefficient between BMI and maximum seonry blokc level was the greatest. It would be clinically useful if the level of spinal anesthesia could be predicted and the 
incidence of side effects could be reduced with only simple body measurements in parturients undergoing caesarean section with spinal anesthesia.

\section{Footnotes}

Authors' Contribution: H. K., M, K. and T. K. designed the research study. S. S. and K. L. performed the research. K. K. and M. K. analyzed the data. Y. P. and K. K. contributed to statistical analysis. S. S., Y. P. and H. K. wrote the manuscript. H. K. and T. K. contributed to the drafting of the manuscript and its critical revision for important intellectual content. All authors contributed to editorial changes in the manuscript. All authors read and approved the final manuscript.

\section{Conflict of Interests: There is no conflict of interest.}

Ethical Approval: This study was approved by the Institutional Review Board of Haeundae Paik Hospital (HPIRB2017-09-001-007). http://www.e-irb.com/index.jsp

Funding/Support: This work was supported by grant from Inje University, 2019.

Informed Consent: Informed consents were obtained from all the patients the night before surgery.

\section{References}

1. Ghaffari S, Dehghanpisheh L, Tavakkoli F, Mahmoudi H. The Effect of Spinal versus General Anesthesia on Quality of Life in Women Undergoing Cesarean Delivery on Maternal Request. Cureus. 2018;10(12). e3715. doi:10.7759/cureus.3715. [PubMed:30788204]. [PubMed Central: PMC6373886].

2. Obstetric Anesthesia and the Society. Practice Guidelines for Obstetric Anesthesia: An Updated Report by the American Society of Anesthesiologists Task Force on Obstetric Anesthesia and the Society for Obstetric Anesthesia and Perinatology. Anesthesiology. 2016;124(2):270-300. doi: 10.1097/ALN.0000000000000935. [PubMed: 26580836].

3. Hocking G, Wildsmith JA. Intrathecal drug spread. $\mathrm{Br} J$ Anaesth 2004;93(4):568-78. doi: 10.1093/bja/aeh204. [PubMed: 15220175].

4. Chun R, Baghirzada L, Tiruta C, Kirkpatrick AW. Measurement of intra-abdominal pressure in term pregnancy: a pilot study. Int $J \mathrm{Ob}$ stet Anesth. 2012;21(2):135-9. doi: 10.1016/j.ijoa.2011.10.010. [PubMed: 22326198].

5. van Bogaert LJ. Lumbar lordosis and the spread of subarachnoid hyperbaric 0.5\% bupivacaine at cesarean section. Int J Gynecol Obstet 2000;71(1):65-6. doi:10.1016/s0020-7292(00)00203-4.

6. Butterworth J, Walker FO, Lysak SZ. Pregnancy increases median nerve susceptibility to lidocaine. Anesthesiology. 1990;72(6):962-5. doi: 10.1097/00000542-199006000-00002. [PubMed: 2350034].

7. Baysal PK, Gölboyu BE, Ekinci M, Güden M, Ahiskalioğlu A, Cem Çelik E. Effects of anthropometric measurements on spinal anaesthesia block characteristics and hemodynamics. Medeni Med J.2016;31(1):23-31. doi: 10.5222/MMJ.2016.023.
8. Norris MC. Patient variables and the subarachnoid spread of hyperbaric bupivacaine in the term parturient. Anesthesiology. 1990;72(3):478-82. doi: 10.1097/00000542-199003000-00015. [PubMed: 2310028]

9. Mordecai MM, Brull SJ. Spinal anesthesia. Curr Opin Anaesthesiol. 2005;18(5):527-33. doi: 10.1097/01.aco.0000182556.09809.17. [PubMed: 16534288].

10. Jawan B, Lee JH, Chong ZK, Chang CS. Spread of spinal anaesthesia for caesarean section in singleton and twin pregnancies. BrJ Anaesth. 1993;70(6):639-41. doi: 10.1093/bja/70.6.639. [PubMed: 8329257].

11. Ekelof NP, Jensen E, Poulsen J, Reinstrup P. Weight gain during pregnancy does not influence the spread of spinal analgesia in the term parturient. Acta Anaesthesiol Scand.1997;41(7):884-7. doi: 10.1111/j.13996576.1997.tb04804.x. [PubMed: 9265932].

12. Malbrain ML, De laet I, Van Regenmortel N, Schoonheydt K, Dits H. Can the abdominal perimeter be used as an accurate estimation of intra-abdominal pressure? Crit Care Med. 2009;37(1):316-9. doi: 10.1097/CCM.ob013e318192678e. [PubMed: 19050639].

13. Akpinar E, Bashan I, Bozdemir N, Saatci E. Which is the best anthropometric technique to identify obesity: body mass index, waist circumference or waist-hip ratio? Coll Antropol. 2007;31(2):387-93. [PubMed: 17847914]

14. Bener A, Yousafzai MT, Darwish S, Al-Hamaq AO, Nasralla EA, AbdulGhani M. Obesity index that better predict metabolic syndrome: body mass index, waist circumference, waist hip ratio, or waist height ratio. J Obes. 2013;2013:269038. doi: 10.1155/2013/269038. [PubMed: 24000310]. [PubMed Central: PMC3755383].

15. Lambert DM, Marceau S, Forse RA. Intra-abdominal pressure in the morbidly obese. Obes Surg. 2005;15(9):1225-32. doi: 10.1381/096089205774512546. [PubMed: 16259876].

16. Taivainen $\mathrm{T}$, Tuominen $\mathrm{M}$, Rosenberg $\mathrm{PH}$. Influence of obesity on the spread of spinal analgesia after injection of plain $0.5 \%$ bupivacaine at the L3-4 or L4-5 interspace. BrJ Anaesth.1990;64(5):542-6. doi: 10.1093/bja/64.5.542. [PubMed: 2354091].

17. Lo K, Wong M, Khalechelvam P, Tam W. Waist-to-height ratio, body mass index and waist circumference for screening paediatric cardiometabolic risk factors: a meta-analysis. Obes Rev. 2016;17(12):1258-75. doi: 10.1111/obr.12456. [PubMed: 27452904].

18. Hyderally H. Complications of spinal anesthesia. Mt Sinai J Med. 2002;69(1-2):55-6. [PubMed: 11832972]

19. Loughrey JP, Walsh F, Gardiner J. Prophylactic intravenous bolus ephedrine for elective Caesarean section under spinal anaesthesia. Eur J Anaesthesiol. 2002;19(1):63-8. doi: 10.1017/s0265021502000108. [PubMed: 11915786].

20. Atashkhoei S, Abri R, Naghipour B, Hatami Marandi P, Fazeli Danesh MT. Effect of Glucose Containing Crystalloid Infusion on Maternal Hemodynamic Status After Spinal Anesthesia for Cesarean Section. Anesth Pain Med.2018;8(4). e80184. doi: 10.5812/aapm.80184. [PubMed: 30271752]. [PubMed Central: PMC6150926].

21. Memary E, Mirkheshti A, Jabbari Moghaddam M, Abtahi D, Yaseri M, Kamali F. Comparison of the effects of pre-anesthetic administration of normal saline, ringer and voluven on the spread of sensory block with hyperbaric bupivacaine spinal anesthesia. Anesth Pain Med. 2014;4(2). e17939. doi: 10.5812/aapm.17939. [PubMed: 24977120]. [PubMed Central: PMC4071270].

22. Harten JM, Boyne I, Hannah P, Varveris D, Brown A. Effects of a height and weight adjusted dose of local anaesthetic for spinal anaesthesia for elective Caesarean section. Anaesthesia. 2005;60(4):348-53. doi: 10.1111/j.1365-2044.2005.04113.x. [PubMed: 15766337].

23. Danelli G, Zangrillo A, Nucera D, Giorgi E, Fanelli G, Senatore R, et al. The minimum effective dose of $0.5 \%$ hyperbaric spinal bupivacaine for cesarean section. Minerva Anestesiol. 2001;67(7-8):573-7. [PubMed: 11602876]. 